BULLETIN (New Series) OF THE

AMERICAN MATHEMATICAL SOCIETY

Volume 44, Number 3, July 2007, Pages 331-359

S 0273-0979(07)01163-9

Article electronically published on May 11, 2007

\title{
SYZYGIES, DEGREES, AND CHOICES \\ FROM A LIFE IN MATHEMATICS. RETIRING PRESIDENTIAL ADDRESS
}

\author{
DAVID EISENBUD
}

The people people work with well, seem very queer to us;

The people people rave about, don't seem quite worth the fuss;

The people people choose for friends, your common sense appall;

But the people people marry, are the queerest folks of all.

—Charlotte Gilman and Annette T. Rubinstein

In 1979 I attended a lecture by Joe Harris about varieties of minimal degree. The subject might be described by saying that it's an extension of the notion of a linearly independent set from the context of finite dimensional vector spaces to algebraic geometry or, from another point of view, by the remarkable equivalence

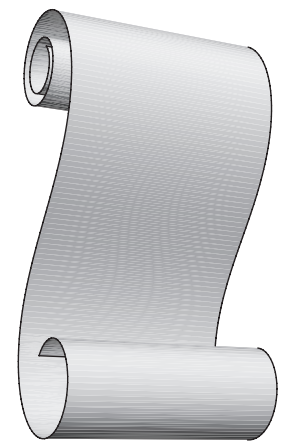

$$
\sim\left(\begin{array}{lllll}
x_{0} & x_{1} & y_{0} & y_{1} & y_{2} \\
x_{1} & x_{2} & y_{1} & y_{2} & y_{3}
\end{array}\right)
$$

FiguRE 1. Scrolls and linear matrices.

I was excited by the mathematics Joe talked about, and the questions we discussed after the talk have interested me ever since. In this report I'll mix a description - meant to be broadly accessible - of the mathematics that grew from this interest with a bit of the personal history that surrounded my involvement.

Mathematically, I'll begin with some elementary algebraic geometry and then explain the first milestone in the theory of varieties of minimal degree, the Del PezzoBertini classification theorem. I'll then describe how Green, Hulek, Popescu and

Received by the editors October 29, 2006, and, in revised form, January 19, 2007.

2000 Mathematics Subject Classification. Primary 14-02; Secondary 14N25, 14Q99, 01-02, $13 \mathrm{D} 02$.

(c) 2007 American Mathematical Society 331 
I came to extend the ideas and the classification from varieties to algebraic sets (unions of varieties). Of course no mathematical subject is ever complete, so I'll finish with a favorite (still unsolved) problem.

Why was this circle of ideas, and not another, so interesting to me? Robin Hartshorne once asked me very earnestly what motivated me to work on a particular problem. At the time I didn't take the question seriously, but I've slowly come to see its importance. How do we form the "mattering map" (in Rebecca Goldstein's wonderful phrase) that tells us what really counts? From the outside, the choice of the problems we fall in love with often seems as irrational as the choice of the people we fall in love with - as in Charlotte Gilman's poem, quoted above.

Of course the hope of fame and glory, the adulation of one's peers, oftenperhaps always - play some role. But to attribute all of our choices to this hope is reductionist, as silly as attributing all of the choice of a marriage partner to sexual attraction. Monetary rewards - sometimes in the form of a tenured job, and even occasionally directly, as with the Clay Mathematics Institute's "million-dollar problems" - are occasionally a factor, but these could hardly be said to have played

a role for me, and I don't think that they do for most mathematicians most of the time.

I'm sure you could make a catalogue of relevant motivations... and then where would you be? As with love and marriage, its usually more interesting to tell stories. So, here goes...

\section{InTRODUCTIONS}

I was a student at the University of Chicago, where I got my $\mathrm{PhD}$ in 1970 . Although parts of the education I got there were very good, I am sorry to say I didn't learn much geometry: when I graduated I could not have computed the homology of a torus (and I certainly couldn't have passed Berkeley's Prelims!). Algebraic geometry was part of this void, but Richard Swan did give one course on it while I was there. He did this for an interesting reason:

My advisor was Saunders Mac Lane, one of the creators of category theory (and much else! [23]). It seemed at the time that algebraic geometry might be entirely swallowed up by category theory. Many ideas leading this way were present in Grothendieck's work, and a big exposition by Demazure and Gabriel [6] appeared near the time I began my studies. Henceforth, algebraic varieties, and indeed all schemes, and of course even very much more, could be thought of as functors. In that period Mac Lane was interested in any application of category theory; he had become a partisan, and perhaps went a little overboard. He talked Richard Swan into giving an introductory course on algebraic geometry from the functorial point of view. Mac Lane himself audited the course. It was understood that I, as Mac Lane's student, would register.

Swan lectured very clearly and very fast, but the course didn't get far: after 11 weeks of hard work Swan was finally able to show that the Grassmannian, the variety of subspaces of a vector space, exists! Not a trace of the beautiful classical geometry nor of the theory of Riemann surfaces and algebraic curves nor of the special examples that are a signature of algebraic geometry was included. Though I've come to understand and value the functorial point of view, I now think it was an awful way to begin learning the subject. 
No, I didn't write a thesis on category theory, but escaped into the domain of noncommutative rings. In a lucky collaboration with a young visitor, Chris Robson, I wrote a thesis somewhat related to what would later become the theory of D-modules (unfortunately I was not visionary enough to imagine the developments that later made that field so hot).

The book of Demazure and Gabriel [6] had another strange effect on my career and on the story here. In my third year of graduate school Mac Lane proposed to take me to my first conference (Category Theory, Battelle, 1968). Looking at the list of lecture series, he suggested that I prepare for and pay special attention to the one of David Buchsbaum on regular local rings.

For some reason I decided that the first chapter of Demazure and Gabriel, which Mac Lane had obtained in manuscript, would be good preparation, but I soon got stuck: the book seemed to be making an elementary logical mistake, leading to the statement that if $I$ is an ideal in a commutative Noetherian ring and if every element of $I$ annihilates some (possibly varying) element of the ring, then all of the elements of $I$ annihilate one fixed element of the ring. Absurd! Well, actually, this is one of the main lemmas in the theory of primary decomposition. I think it was my excitement when I finally untangled the mystery that first hooked me on commutative algebra.

When I got to the conference, Buchsbaum, with his warm personality, solidified my interest and gave it a direction. His lectures were about the dramatic applications of homological algebra to commutative rings that he and Auslander had discovered years before, and the Koszul complex was the center of the action. I wrote up the notes for his talks (now published in the Battelle proceedings; his approach to the Koszul complex can also be found, almost exactly as he presented it, in my book [11]) and enjoyed a personal relationship with Buchsbaum as well. I learned a lot from Buchsbaum's lectures, but the last part of the series was completely obscure to me and remains partly so to this day. The difficulty of understanding only pulled me deeper into the subject.

After I got my PhD I looked for jobs. The pleasure of my relation to Buchsbaum won out easily over an offer from Yale: I took a job at Brandeis.

When I moved to Boston I had an intellectual shock: despite its unity, mathematics looks different from different places. From Chicago you could see that the theory of finite groups was the center of the algebraic world; if you had any doubt, you had only to notice that the seminar on groups lasted twice as long as any other seminar. And you could see that there was no great interest in algebraic geometry. A seminar from Chevalley's extraordinarily ungeometric and by then old-fashioned book was the only presentation of algebraic curves I'm aware of in the four years I was a graduate student there. The view from Boston was inverted: hardly anyone cared about finite groups, but algebraic geometry was at the center of the universe, the subject of several seminars I wanted to follow. So I tried again to learn what algebraic geometry was about.

I listened to a course by Monsky, all valuation theory, à la Weil. But the words in the course didn't seem close to the words in the seminars. I listened to a course by Mumford (it became the book [26]): it was a joy and showed me at last that algebraic geometry might, after all, have something to do with geometry. But I still couldn't understand anything that happened in the seminars. 
Meantime, I began to work seriously in commutative algebra, collaborating with Buchsbaum on the theory of free resolutions and with Graham Evans on things related to the foundations of K-theory. Whereas my knowledge of commutative algebra up to that point was mostly passive, like being able to read a foreign language, these collaborations made me fluent. Buchsbaum was at that time particularly interested in determinantal ideals. He had guessed, many years before, that Macaulay's unmixedness theorem could be strengthened to the assertion that the minors of any size of a generic matrix would be "perfect" - that is, the polynomial ring modulo such an ideal would be Cohen-Macaulay. He wanted to prove this by exhibiting a free resolution of the determinantal ideal with the "right" length and then invoking the Auslander-Buchsbaum formula relating depth and projective dimension. As a student in Kaplansky's classes in Chicago, I had already been fascinated by the potential of homological notions in commutative and noncommutative algebra: one of my earliest papers [8] contains a proof I'm still proud of, a use of the homological tools based on little more than this fascination. I liked the concreteness that free resolutions gave to these homological notions, and I was infected by Buchsbaum's interest in determinantal ideals, too.

Thus, when the book of Hartshorne 21 appeared, I was well-prepared for the algebra it uses. (I think the algebraic requirements are what makes it difficult for many beginners. I don't recommend such a roundabout path to reading it as I took, but it was effective.) I read the book from cover to cover in a wonderful summer month on Martha's Vineyard and did all the exercises 1 At last I could understand the language of the seminars and follow some of the algebraic geometry around me.

The story I want to tell begins with a few notions that appear in the first chapter of Hartshorne's book: the decomposition of an algebraic set into irreducible varieties, the basic notions of dimension and degree, and a linear independence property that can be seen as an elementary part of Bertini's Theorem on hyperplane sections. I'll begin by explaining these.

Ideals and Algebraic Sets. In this paper we'll always work inside a complex projective space $\mathbf{P}_{\mathbf{C}}^{r}=\mathbf{P}^{r}$, which may be thought of as the space of one-dimensional complex subspaces of the vector space $\mathbf{C}^{r+1}$. (We work over $\mathbf{C}$ for the sake of geometric intuition; for the story told here, the shift to an arbitrary field requires only technical changes.) A point $p \in \mathbf{P}^{r}$, as a subspace of $\mathbf{C}^{r+1}$, may be specified by giving the coordinates $\left(x_{0}, \ldots, x_{r}\right)$ of any nonzero vector it contains. We call these homogeneous coordinates of $p$.

If $F\left(x_{0}, \ldots, x_{r}\right)$ is a polynomial and $p \in \mathbf{P}^{r}$ is a point with homogeneous coordinates $\left(a_{0}, \ldots, a_{r}\right)$, then we cannot define $F(p)$, since $F\left(a_{0}, \ldots, a_{r}\right)$ depends on the choice of homogeneous coordinates and not just on $p$. But if $F$ is homogeneous in the sense that all the terms of $F$ have the same degree, then at least it makes sense to say whether $F$ vanishes at $p$, since the values of $F$ at two different sets of homogeneous coordinates differ by a nonzero scalar.

\footnotetext{
${ }^{1}$ Memory is very selective: the other things from that summer that remain sharp in my mind are the work I did on a paper with DeConcini and Procesi; listening over and over to the Brahms Violin Sonatas, which I'd just "discovered"; and rocking my colicky infant daughter. Those who have had a colicky baby will be able to judge what excitement I had from Hartshorne, by the fact that it could compete in intensity of experience.
} 
The objects we are interested in are algebraic sets in $\mathbf{P}^{r}$, that is, sets determined by the vanishing of homogeneous polynomials in the coordinates $x_{i}$. To any algebraic set $X$ we associate the ideal $I(X) \subset S=\mathbf{C}\left[x_{0}, \ldots, x_{r}\right]$ generated by the homogeneous polynomials vanishing at all the points of $X$.

As a topological space, $\mathbf{P}^{r}$ is the quotient of a real $2 r+1$-dimensional sphere (take any point on the sphere, thought of in $\mathbf{C}^{r+1}=\mathbf{R}^{2 r+2}$, to the complex line through the origin and that point). Thus $\mathbf{P}^{r}$ is a compact space. Algebraic sets inside $\mathbf{P}^{r}$ are closed, and thus they are compact too.

Varieties: Dimension and Degree. We call an algebraic set an algebraic variety, or say it is irreducible, if it cannot be written as the union of two smaller algebraic sets. An algebraic set can be written uniquely as a finite union of algebraic varieties - its components - that do not contain each other. This is the simplest and most useful part of the theory called primary decomposition. Figure 2 shows an algebraic set with 5 components.

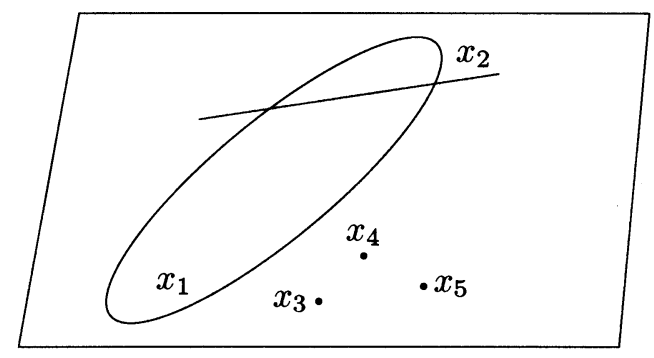

FiguRE 2. An algebraic set with several components of different dimensions.

Any variety $X$ contains a dense open set that is a connected complex analytic submanifold of $\mathbf{P}^{r}$; its complement is an algebraic set of smaller dimension, called the singular locus of $X$ (see Figure 3). It follows that $X$ has a well-defined dimension, the (complex) dimension of this manifold. By definition, $X$ is not the union of finitely many proper subvarieties; it is not hard to prove the stronger statement that any proper subvariety of $X$ has strictly smaller dimension.

For example, a variety has dimension 0 if and only if it consists of a single point. The projective space $\mathbf{P}^{r}$ itself is a variety of dimension $r$. We will see lots more examples later.

An important consequence of compactness is that a variety $X$ of dimension $d$ in $\mathbf{P}^{r}$ meets a general linear space $L$ of complementary dimension $r-d$ in a finite, nonempty set of points whose cardinality is independent of the general space $L$ chosen. This number is called the degree of $X$. More is true: any linear space of dimension $r-d$ that meets $X$ in a finite set meets it in the same number of points when counted with appropriate multiplicities. This was originally proven by elimination theory; we will explain it by algebraic topology (the relevant topological theory was invented explicitly to model some of what was known, via elimination theory, in algebraic geometry). But first, here is a direct argument in case $X \subset \mathbf{P}^{r}$ is a hypersurface, that is, when $X$ is defined by the vanishing of a single nonzero irreducible homogeneous form $F$. For this we need only the Fundamental Theorem of Algebra. 


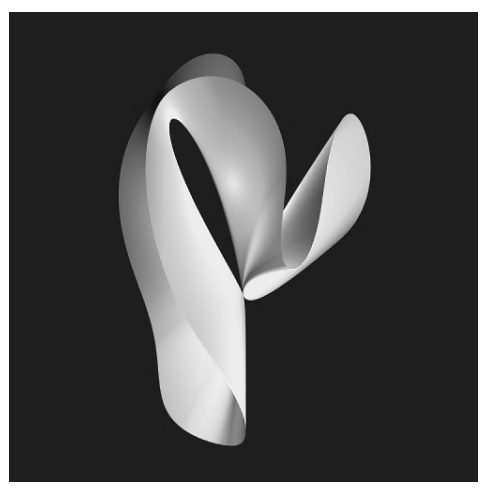

Figure 3 . The variety $\left(x^{2}-y^{3}\right)^{2}=\left(x+y^{2}\right) z^{3}$. Note that the smooth points are dense and form a connected manifold. Over the complex numbers this is always true. Thanks to Herwig Hauser for the picture; see 22 .

The dimension of any hypersurface is $r-1$. Thus to compute the degree of $X$, we must intersect $X$ with a line $L$ not contained in $X$. According to the result above, any such line should have the same number of intersections with $X$ when the intersections are counted with appropriate multiplicities. Suppose that the degree of the defining equation $F$ is $d$. We will show that if $L$ is any line not contained in $X$, then $X$ meets $L$ in $d$ points when counted with multiplicities.

To make the argument explicit we may change coordinates and assume that $L$ is the line defined by the vanishing of the coordinates $x_{2}, \ldots, x_{r}$ and that the point $p:=(1,0, \ldots, 0) \in L$ is not in $X$. The restriction $F\left(x_{0}, x_{1}, 0, \ldots, 0\right)$ of $F$ to $L$ is a sum of monomials of degree $d$ in $x_{0}, x_{1}$. All these monomials except $x_{0}^{d}$ vanish at $p$, so $F$ must contain the monomial $x_{0}^{d}$ with a nonzero coefficient. It follows that the polynomial $f\left(x_{0}\right)=F\left(x_{0}, 1,0, \ldots, 0\right)$ has degree exactly $d$. Since the point $p$ is not in $X$ and every other point of $L$ can be represented in the form $(a, 1,0, \ldots, 0)$, the points of intersection of $L$ with $X$ are the points $(a, 1,0, \ldots, 0)$ such that $f(a)=0$. By the Fundamental Theorem of Algebra, there are $d$ of these, counted with the multiplicities with which they occur as roots of $f$.

To show that for a general line the multiplicities are all equal to 1 requires further argument. Algebraically, it amounts to the remarkable statement that the "discriminant locus", defined to be the set of degree $d$ polynomials $f$ having multiple roots, cannot contain the family of polynomials $f$ that come from restrictions of a form $F$ to lines in $\mathbf{P}^{r}$. Geometrically, it says that the general line in $\mathbf{P}^{r}$ is transverse to $X$. One can prove it by showing that the family of lines that are not transverse to $X$, which is the union of the lines meeting $X$ at singular points and the lines tangent to $X$ at some nonsingular point, is itself an algebraic set, of dimension less than the family of all lines in $\mathbf{P}^{r}$.

Returning to the general case, we give a topological description of the degree of a variety $X$. Any variety has a finite triangulation, and the dense open subset on which it fails to be a submanifold of $\mathbf{P}^{r}$ has real codimension at least two. Suppose that the complex codimension of $X$ in $\mathbf{P}^{r}$ is $c=r-\operatorname{dim} X$. It follows that $X$ has a fundamental class in $\mathrm{H}^{2 c}\left(\mathbf{P}^{r} ; \mathbf{Z}\right)$. The cohomology ring of $\mathbf{P}^{r}$ is $\mathrm{H}^{*}\left(\mathbf{P}^{r} ; \mathbf{Z}\right)=$ $\mathbf{Z}[h] / h^{r+1}$, where $h \in \mathrm{H}^{2}\left(\mathbf{P}^{r}, \mathbf{Z}\right)$ is the fundamental class of a hyperplane and $h^{r}$ is 
the class of a point. It follows that the cohomology class of $X$ may be written in the form $[X]=d \cdot h^{c}$ for some integer $d$.

The $(r-c)$-th power $h^{r-c}$ is the class of any linear subspace $L \subset \mathbf{P}^{r}$ of dimension $r-c$. Thus the set $L \cap X$ supports the class

$$
[L] \cdot[X]=h^{r-c} \cdot d \cdot h^{c}=d \cdot h^{r} .
$$

From the fact that the field of complex numbers is canonically orientable as a real manifold (take 1 first, then $i$ ), it follows that if the two complex varieties of complementary dimension meet in a point, then the multiplicity of intersection at that point must be strictly positive (and is 1 if the varieties meet transversely). We deduce that the number of points of $L \cap X$ is at most $d$, with equality if we count the points with appropriate strictly positive multiplicities. In case $L$ is general, a transversality argument shows that the number of intersection points is exactly $d$. That is, the integer $d$, a cohomological invariant of the embedding of $X$ in $\mathbf{P}^{r}$, is the degree of $X$ as defined above.

More about Plane Sections. We have just explained why a sufficiently general plane $L$ of complementary dimension to a variety $X$ meets $X$ in a number of points that are independent of the plane. But a set of points in $L$ has more structure than just cardinality: the position of the points relative to each other could be interesting, and the simplest questions have to do with linear independence. For example, assuming that the dimension of $L$ is at least 2, could it nevertheless be the case that three or more of the points must lie on a line, no matter which plane is chosen?

Such questions turn out to be subtle and important. In our setting of varieties over $\mathbf{C}$, one can show that for general $L$, in a precise sense, no subset of the points of $X \cap L$ can be geometrically distinguished from another subset of the same cardinality, and it follows with a little argument that no three points of a general hyperplane section can be colinear unless $X$ is a line or a hypersurface. But even this assertion fails for varieties over a field of characteristic $p>0$. (For what's known in characteristic $p$, see Rathmann [28. There are plenty of open problems left there.)

The simplest statement of this kind is important for our story, and we will prove it below. If $X \in \mathbf{P}^{r}$ is an algebraic set, we define the span of $X$ to be the linear space, that is the intersection of all linear spaces containing $X$. The set $X$ is said to be nondegenerate if $X$ spans $\mathbf{P}^{r}$.

Theorem 1 (Linear independence of a plane section). If $X \subset \mathbf{P}^{r}$ is a variety, and if $L$ is a general plane inside $\operatorname{span} X$ with $\operatorname{dim} L=\operatorname{dim} \operatorname{span} X-\operatorname{dim} X$, then the points of $X \cap L$ span $L$.

Since it takes $d$ points to span a space of dimension $d-1$, we immediately get a basic inequality:

Corollary 2. If $X \subset \mathbf{P}^{r}$ is a variety, then $\operatorname{deg} X \geq 1+\operatorname{dim} \operatorname{span} X-\operatorname{dim} X$. For example, if $X$ spans $\mathbf{P}^{r}$, then $\operatorname{deg} X \geq 1+\operatorname{codim} X$.

It's easy to see why Theorem 1 "should" be true: Suppose the points of $X \cap L$ were contained in a plane $L_{0} \subset L$ of dimension one less than $L$. If $p \in X$ is any point not in $L_{0}$, then the span $L^{\prime}$ of $L_{0}$ and $p$ would be a plane of the same dimension as $L$ but would meet $X$ in at least one more point than $L$ does. This seems to 
contradict our assertion, supported by topology, that the number of points in such an intersection is an invariant of $X$, the degree!

There is an obstacle to this line of argument: the awkward possibility that $L^{\prime}$ might meet $X$ in an infinite number of points. Though this obstacle can be circumvented, we will give a different proof, which leads toward an appreciation of the "boundary" case, when the points of $X \cap L$ are linearly independent.

Proof of Theorem 1. We may begin by replacing $\mathbf{P}^{r}$ by the linear span of $X$. Thus we may harmlessly assume that $X$ is nondegenerate, in the sense that $X$ spans $\mathbf{P}^{r}$. We do induction on the quantity

$$
\delta(X):=1+\operatorname{dim} \operatorname{span} X-\operatorname{dim} X .
$$

The number $\delta(X)$ is 1 if and only if $X$ is a linear space. But if $X$ is a linear space (whether or not $X$ is nondegenerate), a complementary linear space meets $X$ in a single point, and the result is obvious.

Suppose now that $\delta(X)>1$, so $X$ is not a linear space. It follows that there is a pair of points $p, q \in X$ such that $X$ does not contain the line $L$ joining $p$ and $q$. Since it is an algebraic condition for the line joining a pair of points $p, q \in \mathbf{P}^{r}$ to lie in $X$, the variety $X$ does not contain the line through a general pair $p, q \in X$.

The lines through $p$ themselves form a projective space of dimension $r-1$. Since not every line through $p$ and another point $q \in X$ lies in $X$, we deduce that the general such line does not lie in $X$ but meets $X$ in only finitely many points.

Let $H \cong \mathbf{P}^{r-1}$ be a hyperplane in $\mathbf{P}^{r}$, and let $\pi_{p}$ be the projection from $p$ to $H$ : this is the map taking a point $q \in X$ to the point $\overline{p, q} \cap H$. Set $Y=\pi_{p}(X)$.

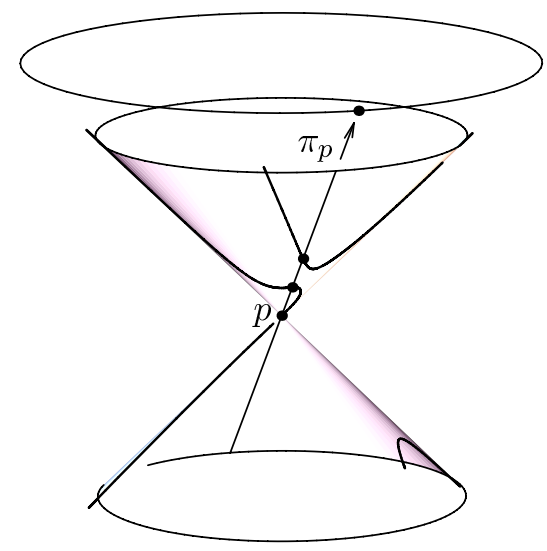

Figure 4. Projection $\pi_{p}$ from a point on a quintic space curve maps the curve two-to-one onto a conic plane curve.

By what we have just proven, the general fiber of $\pi_{p}: X \rightarrow Y$ is finite, and it follows (for example from Sard's Theorem) that $\operatorname{dim} Y=\operatorname{dim} X$. Further, $Y$ is irreducible, because any decomposition of $Y$ into smaller algebraic sets would pull back to a similar decomposition of $X$.

Since $\operatorname{span} X=\mathbf{P}^{r}$ we get $\pi_{p}(\operatorname{span} X)=\mathbf{P}^{r-1}$. A moment's thought shows that $\operatorname{span}\left(\pi_{p} X\right)=\pi_{p}(\operatorname{span}(X \backslash\{p\})$. Because $X$ is irreducible, $X \backslash\{p\}$ is dense in $X$, and it follows that $\operatorname{span}\left(\pi_{p} X\right)=\mathbf{P}^{r-1}$. 
We see now that

$$
\delta(X)=1+\operatorname{dim} \operatorname{span} X-\operatorname{dim} X=1+(1+\operatorname{dim} \operatorname{span} Y-\operatorname{dim} Y)=1+\delta(Y) .
$$

If $M \subset \mathbf{P}^{r-1}$ is a general plane of dimension $(r-1)-\operatorname{dim} Y$, then, by induction, the points of $M \cap Y \operatorname{span} M$. Let $L$ be the linear space $\overline{M, p} \subset \mathbf{P}^{r}$ so that $\operatorname{dim} L=\operatorname{dim} M+1=r-\operatorname{dim} X$. Since $L$ is a general plane through a general point of $X$, it is in fact a general plane of this dimension, and $L$ is visibly spanned by the points of $L \cap X$.

Reflections on Theorem 1. The conclusion of Theorem 1 is often false for algebraic sets. For example, take $X \subset \mathbf{P}^{2}$ to be a line together with a cloud of 1000 points. The dimension of $X$ is 1 , so a plane of complementary dimension is a line $L$. But if $L$ is general, then $L \cap X$ is a single point, so it does not span $L$. The algebraic set in this example has components of different dimensions, but we can also make equidimensional examples: if $X$ is the union of two skew lines in $\mathbf{P}^{3}$, then $X$ spans $\mathbf{P}^{3}$, but the general 2-plane section $L \cap X$ consists of just two points, which cannot span the 2-plane $L$.

In the case where $X$ is a zero-dimensional algebraic set (that is, a finite set of points), the span of $X$ is itself a plane of complementary dimension, and Theorem 1 becomes the tautological statement that the span of $X$ is the span of $X$. In this setting the study of the case where the points not only span but are linearly independent as well is precisely the theory of bases of a vector space. Thus it is tempting to ask whether there's anything special about the algebraic sets $X$ for which the plane section in Theorem 1 is a linearly independent set of points. In general, not much: for example, if we again take $X \subset \mathbf{P}^{2}$ to be a line together with 1000 points and $L$ is a general line, then $L \cap X$ is one point (linearly independent), but this doesn't tell us much about $X$.

On the other hand, in the case where $X$ is irreducible, this independence condition leads to a strong theory. To see why, we can use a result suggested by a careful look at Figure 4, which suggests the proof of the following result:

Lemma 3 (Degree equality). If $X$ is an algebraic variety in $\mathbf{P}^{r}$ and $p \in X$ is a point such that $X$ is not a cone over $p$, then

$$
\operatorname{deg} X=1+\operatorname{deg}\left(\left.\pi_{p}\right|_{X}\right) \cdot \operatorname{deg} \pi_{p} X .
$$

When the points of $L \cap X$ in Theorem 1 are linearly independent so that $\operatorname{deg} X=$ $1+\operatorname{dim} \operatorname{span} X-\operatorname{dim} X$, then by Lemma 3 the same will be true for $\pi_{p}(X)$ and $\operatorname{deg}\left(\left.\pi_{p}\right|_{X}\right)=1$. Following the induction down to the case $1+\operatorname{dim} \operatorname{span} X-\operatorname{dim} X=1$ where $X$ is a linear space, we see that $X$ admits a degree 1 map to a linear space, and thus a dense open set of $X$ is isomorphic to a dense open set of a linear space; in the language of algebraic geometry, $X$ is rational. But rationality is just the tip of the iceberg - the varieties $X$ for which $L \cap X$ is linearly independent are more special yet. This brings me to the next chapter in my story.

\section{VARIETIES OF Minimal DEGREe}

In 1978, with new-found confidence, I faithfully attended the algebraic geometry seminars at Harvard and MIT. Zariski, born in 1899, was still in the audience at Harvard, heckling the speakers; he was older than the next oldest regulars-Artin, 
Kleiman, and Mumford-by about 35 years. G. H. Hardy's famous assertion that all significant research is done by the young was obviously not respected by Zariski 2

At the MIT seminar, Artin and Kleiman were the ringleaders. One day the speaker there was a young instructor, Joe Harris. His topic was something I could warm to as an algebraist: the question of whether the ideals of certain curves in projective space could be generated by quadrics of rank 4, that is, polynomials of the form $p q-r s$ where $p, q, r, s$ are linear forms. He had the good grace, unusual in any speaker but most unusual in the very young, to give love and attention even to the ancient and elementary tool he needed, the theory of varieties of minimal degree. I'm sure that those of you who have had the pleasure of hearing Harris lecture will know what I mean - his open style hasn't really changed in the 25 years I've been listening to him.

Classical as Harris' subject was, it was all news to me. But my hope of contributing something was immediately awoken: Varieties of minimal degree are closely related to the determinantal ideals I'd already studied. Let me tell you a little about them.

When the spanning set of points in Theorem 1 is also linearly independent, we will say that $X$ is a variety of minimal degree. The name comes from the reformulation in terms of Corollary 2 2 since the points of the plane section $X \cap L$ span $L$, minimal degree means that the inequality

$$
\operatorname{deg} X \geq \operatorname{dim} \operatorname{span} X-\operatorname{dim} X+1
$$

in Corollary 2 is an equality.

A linear subspace $X \subset \mathbf{P}^{r}$ of any dimension $d$ is an easy example: $X$ has degree 1 and is its own linear span, so the inequality $(*)$ has the form $1 \geq d-d+1$. Only slightly less trivial is the conic in the plane. The linear span is the whole plane, and the degree ( $=$ number of times the conic meets a line) is 2 , so $(*)$ becomes $2 \geq 2-1+1$ : the conic is a variety of minimal degree.

We can immediately generalize to any quadric hypersurface: the variety in $\mathbf{P}^{r}$ defined by the vanishing of a single quadratic form has dimension $r-1$ and degree $2=r-(r-1)+1$, so every quadric is a variety of minimal degree.

Veronese Embeddings. A different way of looking at the conic yields another important family of examples. The familiar parabola $x_{2}=x_{1}^{2}$ can be parametrized by the formulas $x_{1}=t$ and $x_{2}=t^{2}$. To move to projective coordinates we homogenize the equation $x_{2}-x_{1}^{2}=0$ to get $x_{0} x_{2}-x_{1}^{2}=0$, and the parametrization becomes

$$
x_{0}=s^{2}, \quad x_{1}=s t, \quad x_{2}=t^{2} .
$$

One should think of these formulas as defining a morphism

$$
\phi: \mathbf{P}^{1} \rightarrow \mathbf{P}^{2} \quad: \quad(s, t) \mapsto\left(s^{2}, s t, t^{2}\right) .
$$

Instead of using the monomials of degree 2 in $s$ and $t$, we can generalize and use the monomials of any degree, $d$. We get

$$
\phi: \mathbf{P}^{1} \rightarrow \mathbf{P}^{d} \quad: \quad(s, t) \mapsto\left(s^{d}, s^{d-1} t, \ldots, t^{d}\right) .
$$

The image of $\phi$ is a curve in $\mathbf{P}^{d}$ called the rational normal curve of degree $d$, or rational normal $d$-ic curve. We will write $\Sigma(d)$ for this curve.

\footnotetext{
${ }^{2}$ Though Hardy's proposition contains some truth, I think it is partly self-fulfilling. I believe that fewer people would subscribe to it now than in 1970, and the distribution of ages at research seminars seems to me more uniform.
} 
To compute the degree of $\Sigma(d)$, we must count the points of intersection with a general hyperplane $\sum_{i} a_{i} x_{i}=0$. It's easiest to perform the count back on the source $\mathbf{P}^{1}$ of the morphism $\phi$. Thinking of $\phi$ as an inclusion, the restriction to $\mathbf{P}^{1}$ of the linear form $x_{i}$ is the monomial $s^{d-i} t^{i}$, so $\sum_{i} a_{i} x_{i}$ pulls back to a general form of degree $d$ on $\mathbf{P}^{1}$. Any nonzero form of degree $d$ on $\mathbf{P}^{1}$ has exactly $d$ zeros counting multiplicities (if we de-homogenize and regard our form of degree $d$ as coming from a polynomial of degree $d$ in one variable, this is just the Fundamental Theorem of Algebra). For a general form the zeros are distinct, so the degree of $\Sigma(d)$ is $d$.

We are now in a position to see whether $\Sigma(d)$ is a variety of minimal degree. The formula above shows that a nonzero linear form on $\mathbf{P}^{d}$ restricts to a nonzero form on $\mathbf{P}^{1}$, so no linear form vanishes identically on $\Sigma(d)$ : that is, the span of $\Sigma(d)$ is all of $\mathbf{P}^{d}$. The inequality $(*)$ becomes

$$
d \geq d-1+1 .
$$

Since we have equality, $\Sigma(d)$ has minimal degree.

It is natural to try to generalize to maps $\mathbf{P}^{n} \rightarrow \mathbf{P}^{r}$. The "next" example in this way of thinking is the map

$$
\nu: \mathbf{P}^{2} \rightarrow \mathbf{P}^{5} \quad: \quad(s, t, u) \mapsto\left(s^{2}, s t, t^{2}, s u, t u, u^{2}\right),
$$

whose image is called the Veronese surface in $\mathbf{P}^{5}$.

We can compute the degree of the Veronese surface $X$ almost as easily as that of the rational normal $d$-ic curve. This time we must choose a general 3-plane and count the number of points of the intersection with $X$. A 3 -plane in $\mathbf{P}^{5}$ may be written as the intersection of two hyperplanes, say $H_{1}$ and $H_{2}$, so we must count the points of $X \cap H_{1} \cap H_{2}$. Each $H_{i}$ is defined by the vanishing of a linear form on $\mathbf{P}^{5}$, say $\ell_{i}=\sum a_{i, j} x_{i}=0$. Since the $x_{i}$ restrict to the quadratic monomials on $\mathbf{P}^{2}$, the $\ell_{i}$ pull back to two arbitrary nonzero quadratic forms on $\mathbf{P}^{2}$, and any two quadrics occur this way. Thus the points of $X \cap H_{i}$ pull back to the points of a conic on $\mathbf{P}^{2}$, and the degree of $X$ is nothing but the number of intersection points of two general conics, that is, $\operatorname{deg} X=4$. (We have used an easy special case of Bézout's Theorem: distinct irreducible curves of degree $d$ and $e$ in the complex projective plane meet in de points, counting multiplicity, as in Figure 5.)

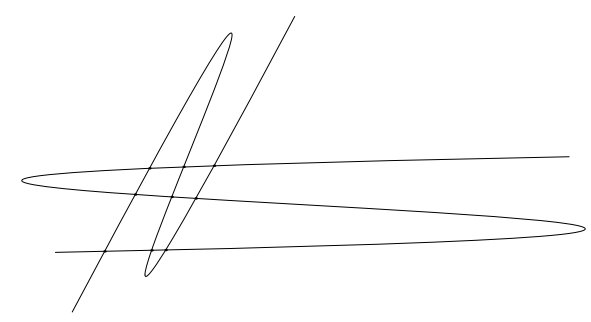

Figure 5. Two cubic plane curves meet in 9 points.

Since the quadratic monomials used to define $\nu$ are linearly independent, $X$ spans all of $\mathbf{P}^{5}$, and the inequality $(*)$ becomes

$$
4 \geq 5-2+1=4
$$

since this is an equality, the Veronese surface has minimal degree. 
Continuing in this vein, we could next try the cubic Veronese surface defined as the image of the map using all the 10 degree 3 monomials on $\mathbf{P}^{2}$,

$$
\nu: \mathbf{P}^{2} \rightarrow \mathbf{P}^{9} \quad: \quad(s, t, u) \mapsto\left(s^{3}, s^{2} t, \ldots u^{3}\right) .
$$

Arguing exactly as before, we see that the degree of the cubic Veronese surface $Y \subset \mathbf{P}^{9}$ is the number of points of intersection of two cubic curves in the plane, that is, $\operatorname{deg} Y=9$. The inequality $(*)$ becomes

$$
9 \geq 9-2+1=8 .
$$

But this time, the inequality is strict: the cubic Veronese surface is not a variety of minimal degree!

Instead of looking at the cubic Veronese surface, we could try the quadric Veronese 3 -fold, given by the ten monomials of degree 3 in four variables,

$$
\mathbf{P}^{3} \rightarrow \mathbf{P}^{9} \quad: \quad(s, t, u, v) \mapsto\left(s^{2}, s t, \ldots v^{2}\right),
$$

or indeed the $d$-ic Veronese $n$-fold, given by all forms of degree $d$ in $n+1$ variables. But a little calculation shows that none of these examples is a variety of minimal degree except the rational normal curves and the Veronese surface in $\mathbf{P}^{5}$. This generalization has reached a dead end!

Rational Normal Scrolls. The examples of varieties of minimal degree provided by the family of rational normal curves, and by the Veronese surface in $\mathbf{P}^{5}$, were inspired by our understanding of plane conic curves. Neither of these examples includes the nonsingular quadric surface in $\mathbf{P}^{3}$. To produce more examples we can take inspiration from one of its many classical geometric descriptions, illustrated in Figure 66 Start with two lines $L_{1}$ and $L_{2}$ in $\mathbf{P}^{3}$ that do not meet each other. Choose an isomorphism between them; to make this symmetric, think of it as a pair of embeddings $\phi_{1}$ and $\phi_{2}$ of $\mathbf{P}^{1}$ in $\mathbf{P}^{3}$, with images $L_{1}$ and $L_{2}$. The union of

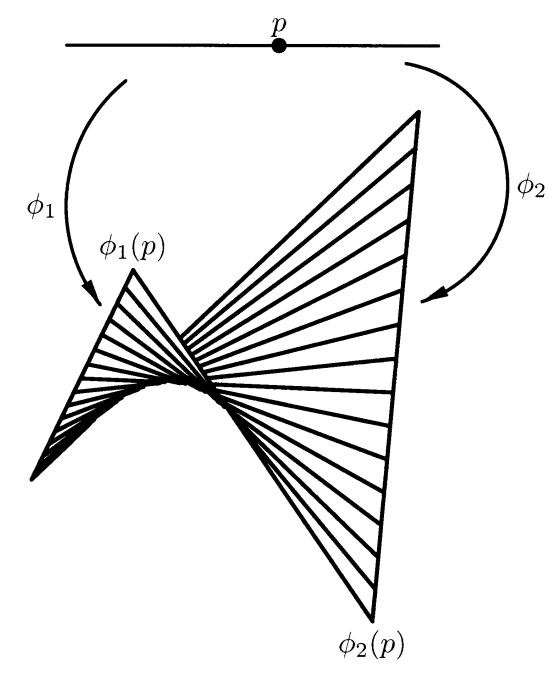

FiguRE 6. Smooth quadric surface as the union of lines joining corresponding points on two skew lines in 3-space. 
the lines joining pairs of points $\phi_{1}(p)$ and $\phi_{2}(p)$, that is,

$$
\Sigma=\bigcup_{p \in \mathbf{P}^{1}} \overline{\phi_{1}(p), \phi_{2}(p)}
$$

is a surface in $\mathbf{P}^{3}$.

To understand the surface $\Sigma$ algebraically, choose coordinates $x, y, u, v$ on $\mathbf{P}^{3}$ so that $L_{1}$ is given by the equations $x=y=0$ and $L_{2}$ is given by $u=v=0$. When we have done this, we may treat $u, v$ as coordinates on the line $L_{1}$, and $x, y$ as coordinates on $L_{2}$. We can further adjust the choice so that isomorphism between $L_{1}$ and $L_{2}$ is the identity in these coordinates.

If the point $\phi_{1}(p)$ is given by the equations $a u+b v=0$ on $L_{1}$, then $\phi_{2}(p)$ on $L_{2}$ has equations $a x+b y=0$ on $L_{2}$. The line $L_{p}=\overline{\phi_{1}(p), \phi_{2}(p)}$ has equations

$$
a u+b v=0, \quad a x+b y=0
$$

in $\mathbf{P}^{3}$.

To find the equation of the surface $\Sigma$, we must produce a form that vanishes on the points of all these lines. We could do this by elimination theory, but there is a trick that produces the answer directly. Consider the matrix

$$
M=\left(\begin{array}{ll}
u & x \\
v & y
\end{array}\right) .
$$

If we take $a$ times the first row $(u, x)$ and add $b$ times the second row $(v, y)$, we get the generalized row $(a u+b v, a x+b y)$ whose entries are the linear forms vanishing on $L_{p}$. Since the entries $a u+b v$ and $a x+b y$ of this linear combination of rows vanish on $L_{p}$, the determinant $Q=u y-v x$ of $M$ must vanish on $L_{p}$ too. Thus $Q$ is in the ideal of $\Sigma$. It is easy to see directly that $Q$ is an irreducible polynomial, so $Q$ generates the ideal of $\Sigma$; that is, $\Sigma$ is a quadric surface in $\mathbf{P}^{3}$. Since the four partial derivatives of $Q$ generate the maximal homogeneous ideal, $\Sigma$ is nonsingular. Up to a linear change of coordinates, the only invariant of a quadratic form over the complex numbers is its rank, so we may even say that $\Sigma$ is the nonsingular quadric in $\mathbf{P}^{3}$.

We can try to generalize the construction of the quadric given above by replacing the straight lines $L_{1}, L_{2}$ with curves $C_{1}, C_{2} \subset \mathbf{P}^{r}$ that are images of a common curve $C$ under embeddings $\phi_{i}: C \rightarrow \mathbf{P}^{r}$. Let us assume for simplicity that the spans of the $C_{i}$ are disjoint linear subspaces $L_{1}, L_{2}$ of dimensions $e_{1}, e_{2}$ in $\mathbf{P}^{r}$. We could represent the surface

$$
\Delta=\bigcup_{p \in C} \overline{\phi_{1}(p), \phi_{2}(p)}
$$

by the picture in Figure 7 .

To compute the degree of $\Delta$ we must compute how many points it shares with a general codimension 2 linear space in $\mathbf{P}^{r}$. Since the codimension 2 plane can be written as the intersection of two hyperplanes, the degree of $\Delta$ can also be computed as the degree of a curve obtained by intersecting $\Delta$ with a hyperplane. For this purpose, we choose a hyperplane $H$ general among those containing $L_{1}$. Since $H$ meets $L_{2}$ in a linear space that is a general hyperplane inside $L_{2}$, it follows that the intersection $H \cap \Delta$ is the union of the curve $C_{1}$ with $\operatorname{deg} C_{2}$ lines; thus $H \cap \Delta$ is a curve of degree $\operatorname{deg} C_{1}+\operatorname{deg} C_{2}$. Of course we haven't chosen a general hyperplane $H$, but a little care shows that our choice was "general enough" and the degree of $\Delta$ really is $\operatorname{deg} C_{1}+\operatorname{deg} C_{2}$. 


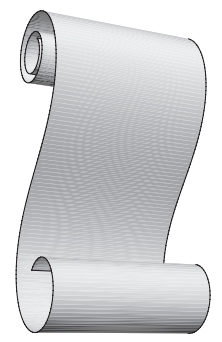

Figure 7 . The union of a family of straight lines.

On the other hand, the span of $\Delta$ is the same as the span of $L_{1} \cup L_{2}$, so

$$
\operatorname{dim} \operatorname{span} \Delta=1+\operatorname{dim} \operatorname{span} C_{1}+\operatorname{dim} \operatorname{span} C_{2} .
$$

It follows by easy arithmetic that $\Delta$ is a variety of minimal degree if and only if both $C_{1}$ and $C_{2}$ are! Since the only examples of curves of minimal degree that we know are the rational normal curves, we take $C_{i}$ to be $\Sigma\left(d_{i}\right)$ and rename $\Delta=\Sigma\left(d_{1}, d_{2}\right)$.

Consideration of the proof above leads to a still more general family of examples: Let $C_{1}, \ldots, C_{e} \subset \mathbf{P}^{r}$ be rational normal curves whose linear spans $L_{1} \ldots, L_{e}$ are independent in the sense that $L_{i}$ does not meet the linear span of $\cup_{j \neq i} L_{j}$. For each $i$, let $\mathbf{P}^{1} \rightarrow C_{i} \subset \mathbf{P}^{r}$ be a parametrization, and set

$$
\Sigma\left(d_{1}, \ldots, d_{e}\right)=\bigcup_{p \in \mathbf{P}^{1}} \overline{\phi_{1}(p), \ldots, \phi_{e}(p)} .
$$

The variety $\Sigma\left(d_{1}, \ldots, d_{e}\right)$ is called a rational normal scroll. It has dimension $e+1$, and the same kind of argument as before shows, by induction on $e$, that its degree is $\sum_{i} d_{i}$, while the dimension of its linear span is $\left(\sum_{i}\left(d_{i}+1\right)\right)-1$. We can even allow some (but not all) of the $d_{i}$ to be 0 , thinking of the rational normal curve of degree 0 as a point; for example, $\Sigma(1,0, \ldots, 0)$ is a linear space, and in general $\Sigma\left(d_{1} \ldots, d_{e}, 0\right)$ is a cone over $\Sigma\left(d_{1}, \ldots, d_{e}\right)$. Arithmetic now shows:

Proposition 4. Any rational normal scroll is a variety of minimal degree.

Further Examples? We have now found one family of varieties of minimal degree, the rational normal curves and Veronese surface, by generalizing the conic in the plane and another, the rational normal scrolls, by generalizing the quadric surface in $\mathbf{P}^{3}$. Can we continue with the nonsingular quadric in $\mathbf{P}^{4}$ or in some other way to get further new examples? Amazingly, the answer is no. The story was worked out for surfaces by Del Pezzo in [27] and extended to all dimensions by Bertini in [1.

Theorem 5 (Del Pezzo-Bertini). Any variety of minimal degree is, up to a linear transformation, one of the following:

(1) A quadric hypersurface,

(2) A cone over the Veronese in $\mathbf{P}^{5}$,

(3) A rational normal scroll.

Even today, a proof requires some work. One is given, for example, in EisenbudHarris [15]. 
How Scrolls Appear in Nature. Let me digress for a moment to explain why Harris was talking about scrolls in his MIT lecture. This had little to do with the classification theorem, but rather with one of the ways scrolls appear in nature: Suppose $X \subset \mathbf{P}^{r}$ is a variety and that some hyperplane section $H \cap X$ of $X$ is the union of two algebraic sets, say $H \cap X=D \cup E$. For simplicity, suppose that the intersection of $H$ and $X$ is transverse at some point on each component of $D$ and $E$ (otherwise one has to consider scheme-theoretic intersections).

Now suppose that $E$ does not span $H$ - this is a powerful assumption. It follows that we can choose an $(r-2)$-dimensional linear space $L$ containing $E$. Let $H_{\lambda}$ be the family of all hyperplanes in $\mathbf{P}^{r}$ containing $L$, and set

$$
D_{\lambda}=\left(H_{\lambda} \cap X\right) \backslash E .
$$

Theorem 6. In the setting above,

$$
\Sigma=\operatorname{closure}\left(\bigcup_{\lambda} \overline{D_{\lambda}}\right),
$$

the closure of the union of the linear spans of the $D_{\lambda}$ in $\mathbf{P}^{r}$, is a rational normal scroll $\mathrm{3}^{3}$

This construction allows some questions about embeddings of varieties with high codimension in projective space to be reduced to questions of embeddings of small codimension - usually easier - in scrolls. The idea is old, going back at least to the book of Room 29]. The interested reader will find a modern account, with some refinements, in my paper [10].

Example: For an easy example, take the case where $X$ is an irreducible quadric surface - smooth or not-in $\mathbf{P}^{3}$ and $H$ is a plane cutting $X$ in the union of two distinct lines $D$ and $E$. Since neither line spans $H$, we can apply Theorem 6 , In this case, each $D_{\lambda}$ is a line, so $\overline{D_{\lambda}}=D_{\lambda}$ and the scroll in question is just the union of the lines residual to $E$ - that is, $X$ itself, which is the scroll $\Sigma(2,0)$ in the singular case (Fig. 8) or $\Sigma(1,1)$ in the smooth case (Fig. 6).

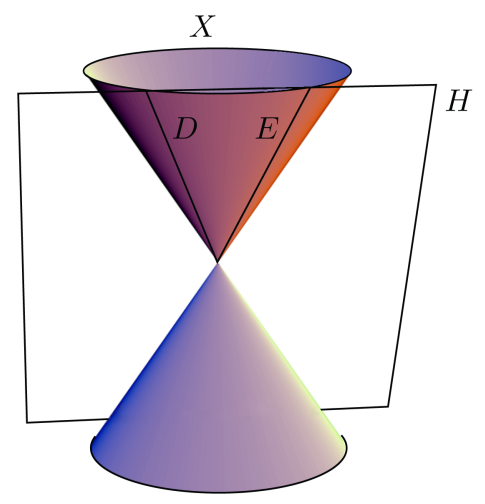

FiguRE 8. A plane through the vertex meets the quadric cone in two lines.

\footnotetext{
${ }^{3}$ The need to take the closure can be eliminated if one works with schemes. This is one of the many ways this apparently ungeometric theory simplifies geometry.
} 
Example: Here is an example I took up as an exercise soon after Harris' lecture (you can see what I was able to make of it in 9.) Take any smooth curve $X \subset \mathbf{P}^{r}$ of genus $g>1$ such that there is a 2-to-1 map $\phi: X \rightarrow \mathbf{P}^{1}$; such curves are called hyperelliptic. We will assume that $r \geq 3$ (in fact, no hyperelliptic curve can be embedded in $\mathbf{P}^{2}$.) Let $D$ be a fiber of $\phi$ that consists of two distinct points. Let $H$ be a hyperplane containing $D$. Since the dimension of $H$ is at least 2, the two points of $D$ cannot span $H$. If we assume that the complement $E=H \cap X \backslash D$ also fails to span $H$, then we see that $X$ lies inside a 2-dimensional scroll in $\mathbf{P}^{2}$. It turns out that this condition is often satisfied.

Example: Let $X \subset \mathbf{P}^{g-1}$ be a smooth, nonhyperelliptic curve of genus $g>1$ in its canonical embedding, and suppose that there is a 3 -to- 1 map from $\phi: X \rightarrow \mathbf{P}^{1}$. Let $D \subset X$ be a set of three points that form a fiber of $\phi$. The Riemann-Roch formula implies that $D$ is contained in a line and that if we choose a general hyperplane $H$ containing $D$, then $E=(H \cap X) \backslash D$ does not span $H$. Thus $X$ is contained in a 2-dimensional rational normal scroll! This is the result for which Harris needed to speak about scrolls.

Example: Yet more examples arise in the study of the canonical maps of varieties of general type; when these are not embeddings, they often turn out to have scrolls as images. The simplest cases are the hyperelliptic curves, whose canonical images are the rational normal curves. See for example [18 for a start on the literature around this subject.

\section{BACK TO MATRICES AND RESOLUTIONS}

By the time I heard Harris's lecture, I'd been working with Buchsbaum on determinantal ideals and their free resolutions for some time. Perhaps the thing that charmed me most of all in Harris's lecture was the appearance of a host of wonderfully special matrices that, despite my previous work, I'd never seen before. These arise when one wants to describe the ideals of forms vanishing on varieties of minimal degree. I now know that they should be considered part of the Kronecker-Weierstrass theory of matrix pencils, which otherwise seems to be mostly the province of applied mathematicians and matrix theorists these days.

First, one can show that the ideal of forms vanishing on the rational normal curve $\Sigma(d)$ of degree d, thought of as embedded in its span $\mathbf{P}^{d}$, is generated by the $2 \times 2$ minors of the matrix

$$
M(d):=\left(\begin{array}{cccc}
x_{0} & x_{1} & \cdots & x_{d-1} \\
x_{1} & x_{2} & \cdots & x_{d}
\end{array}\right)
$$

More generally, the ideal of the scroll $\Sigma\left(d_{1}, \ldots, d_{e}\right)$ is generated by all $2 \times 2$ minors of the matrix $M\left(d_{1}, \ldots, d_{e}\right)$ obtained by simply concatenating the matrices $M\left(d_{i}\right)$,

$$
M\left(d_{1}, \ldots, d_{e}\right)=M\left(d_{1}\right)|\cdots| M\left(d_{e}\right) .
$$

For example, the ideal of the scroll $\Sigma(2,3)$ in $\mathbf{P}^{6}$, obtained geometrically by joining corresponding points on a line and a conic, is generated by the ideal of $2 \times 2$ minors of the matrix

$$
M(2,3)=\left(\begin{array}{lllll}
x_{0} & x_{1} & y_{0} & y_{1} & y_{2} \\
x_{1} & x_{2} & y_{1} & y_{2} & y_{3}
\end{array}\right)
$$

What nice simple patterns the variables in these matrices had, I thought. But there was more: Harris needed the fact that the relations between the minors of these 
matrices, called syzygies, are generated by relations whose coefficients are forms of degree 1 , that is, by linear syzygies.

Here's the simplest example: by what's gone before, the ideal of forms vanishing on the rational normal curve $\Sigma(3)$ of degree 3 in $\mathbf{P}^{3}$ (which is usually called the twisted cubic) is generated by the three $2 \times 2$ minors of the matrix

$$
M(3)=\left(\begin{array}{lll}
x_{0} & x_{1} & x_{2} \\
x_{1} & x_{2} & x_{3}
\end{array}\right) .
$$

One way to get relations among these minors is to use the fact that the $3 \times 3$ matrix we'd get from $M(3)$ by duplicating one of the rows, say

$$
M(3)^{(1)}:=\left(\begin{array}{lll}
x_{0} & x_{1} & x_{2} \\
x_{0} & x_{1} & x_{2} \\
x_{1} & x_{2} & x_{3}
\end{array}\right),
$$

has determinant 0 (it has a repeated row). The determinant can be expanded in terms of the minors of the submatrix consisting of the last two rows as

$$
0=\operatorname{det}\left(M(3)^{(1)}\right)=x_{0} \cdot m_{0}+x_{1} \cdot m_{1}+x_{2} \cdot m_{2}
$$

where $m_{i}$ denotes $(-1)^{i}$ times the determinant of the submatrix obtained from $M(3)$ by omitting the $i$-th column. If we do the same trick but repeat the second row of $M(3)$ instead of the first, we get a second relation, and it can be shown that these generate the module of all relations,

$$
N:=\left\{\left(a_{1}, a_{2}, a_{3}\right) \in S^{3} \mid \sum a_{i} \cdot m_{i}=0\right\},
$$

called the module of (first) syzygies of $\left(m_{1}, m_{2}, m_{3}\right)$. This module of syzygies is linear in the sense that the two generating relations, which are the rows of the matrix $M(3)$, have entries that are linear forms; they are linear syzygies.

The ring $S_{\Sigma(d)}:=S /\left(m_{1}, m_{2}, m_{3}\right)$ is called the homogeneous coordinate ring of the twisted cubic. It is convenient to represent what we've just learned by writing a free resolution of $S_{\Sigma(d)}$, namely the exact sequence

$$
0 \longrightarrow S(-3)^{2} \stackrel{\left(\begin{array}{ll}
x_{0} & x_{1} \\
x_{1} & x_{2} \\
x_{2} & x_{3}
\end{array}\right)}{\longrightarrow} S(-2)^{3} \stackrel{\left(\begin{array}{lll}
m_{1} & m_{2} & m_{3}
\end{array}\right)}{\longrightarrow} S \longrightarrow S_{\Sigma(3)} \longrightarrow 0 .
$$

Here the notation $S(-3)$ denotes the graded free module of rank 1 , shifted in degree to have its generator in degree three 4 and $S(-3)^{2}$ denotes the direct sum of two of these. The shifts in the modules are given so as to make all the maps preserve degrees. For example, the generators of the free module $S(-2)^{3}$ go to the degree two elements $m_{i} \in S$. The linearity of the syzygies is expressed by the fact that the twist -3 of the left-hand free module is one more than the twist -2 of the free module in the middle, so the elements of the matrix representing the map between these two modules are all forms of degree $3-2=1$.

\footnotetext{
${ }^{4}$ One might think this notation should denote a free module with generator in degree -3 ! Here is a version that might make the given choice seem more natural: if $M=\oplus M_{n}$ is a graded module whose degree $n$ component is $M_{n}$ and $d$ is an integer, then $M(d)$ denotes the same module with grading shifted so that $M(d)_{n}=M_{d+n}$.
} 
More Matrices, More Surprises. In the next sections I will talk about a problem that occurred to me very soon after Harris's lecture and will follow my involvement with it. But I can't resist digressing again first to mention some other surprises that grew out of the study of the matrices $M\left(d_{1}, \ldots, d_{e}\right)$. The matrices $M\left(d_{1}, \ldots, d_{e}\right)$ are the beginning of a long and surprising story: for example, the ideal of the Veronese surface $\nu\left(\mathbf{P}^{2}\right) \subset \mathbf{P}^{5}$ is generated by the $2 \times 2$ minors of the generic symmetric matrix

$$
N=\left(\begin{array}{ccc}
x_{0} & x_{1} & x_{2} \\
x_{1} & y & x_{3} \\
x_{2} & x_{3} & x_{4}
\end{array}\right) .
$$

The hyperplane section of the Veronese surface is a curve of degree 4 in $\mathbf{P}^{4}$, thus also a variety of minimal degree. By the classification theorem we see that it must be $\Sigma(4)$. It follows (after a little more work) that the ideal of $2 \times 2$ minors of

$$
M(4)=\left(\begin{array}{llll}
x_{0} & x_{1} & x_{2} & x_{3} \\
x_{1} & x_{2} & x_{3} & x_{4}
\end{array}\right)
$$

is equal to the ideal of $2 \times 2$ minors of the matrix obtained from $N$ above by setting $y=x_{2}$,

$$
\left(\begin{array}{lll}
x_{0} & x_{1} & x_{2} \\
x_{1} & x_{2} & x_{3} \\
x_{2} & x_{3} & x_{4}
\end{array}\right) .
$$

More generally, one can show (Conca [5, Cor. 2.2]) that the ideal of $2 \times 2$ minors of the matrix $M(d)$ is the same as the ideal of $2 \times 2$ minors of any of the Hankel matrix

$$
\left(\begin{array}{cccc}
x_{0} & x_{1} & \cdots & x_{s} \\
x_{1} & x_{2} & \cdots & x_{s+1} \\
x_{2} & x_{3} & \cdots & x_{s+2} \\
\vdots & \vdots & & \vdots \\
x_{d-s} & x_{d-s+1} & \cdots & x_{d}
\end{array}\right) .
$$

All the matrices we've described are of a type I've called 1-generic; see my paper [10] and the references there for further theory and applications.

\section{The EAgOn-NorthCott REsolution}

Harris' remark that the syzygies on the generators of the ideals of the scrolls were always linear struck a chord with me: I'd seen those linear relations before. I thought the things I knew about determinantal ideals from my work with Buchsbaum might be useful; perhaps we algebraists could even teach the geometers something! The idea was intoxicating.

I'll now describe what you need to know to appreciate the information that I had. It is contained in the theory of the Eagon-Northcott complex 7], which is the (minimal) free resolution of the ideal generated by the $p \times p$ minors of a generic $p \times q$ matrix.

There are slick ways to present the Eagon-Northcott complex based on representation theory or exterior algebra; see for example Eisenbud [11] for the exterior algebra path. Unfortunately, they require either a lot of prior comfort with the tools or enough time to become comfortable. However, the idea of the Eagon-Northcott complex can be given simply and directly. Things look simpler still if one sticks to 
the case $p=2$. Since that is the case we will need, we will allow ourselves that simplification.

Let

$$
M=\left(\begin{array}{lll}
u_{1} & \ldots & u_{q} \\
v_{1} & \ldots & v_{q}
\end{array}\right)
$$

be the generic $2 \times q$ matrix over the polynomial $\operatorname{ring} T:=\mathbf{C}\left[u_{1}, \ldots, u_{q}, v_{1}, \ldots, v_{q}\right]$. We regard $T$ as the ring of polynomial functions on the space of $2 \times q$ matrices over C. Let $m_{i, j}=u_{i} v_{j}-u_{j} v_{i}$ be the determinant of the submatrix involving just columns $i$ and $j$. The $m_{i, j}$ all vanish on a given matrix if and only if that matrix has rank 1 , so the ideal $I=\left(\left\{m_{i, j}\right\}\right)$ defines the variety $X$ of $2 \times q$ matrices of rank one. It turns out that $I$ is prime, so $I=I(X)$, the ideal of all forms vanishing on $X$.

There are $\left(\begin{array}{l}q \\ 2\end{array}\right)$ forms $m_{i, j}$, each of degree 2 , so we may begin a graded free resolution of the homogeneous coordinate ring $S_{X}=T / I$ with

$$
T(-2)^{\left(\begin{array}{l}
q \\
2
\end{array}\right)} \rightarrow T \rightarrow T_{X} \rightarrow 0 .
$$

Further, for each triple of distinct indices $\{i<j<k\}$ we can play the trick of the repeated row used to analyze the twisted cubic above, and we get two linear syzygies,

$$
u_{i} m_{j, k}-u_{j} m_{i, k}+u_{k} m_{i, j},
$$

and a second involving the $v$ 's in place of the $u$ 's. It turns out that these "three at a time" syzygies generate all the syzygies on the $m_{i, j}$, so the beginning of the resolution given above can be extended to

$$
T(-3)^{2\left(\begin{array}{l}
q \\
3
\end{array}\right)} \rightarrow T(-2)^{\left(\begin{array}{l}
q \\
2
\end{array}\right)} \rightarrow T \rightarrow T_{X} \rightarrow 0 .
$$

A pattern is clear: one should guess that the next term would be

$$
T(-4)^{3\left(\begin{array}{l}
q \\
4
\end{array}\right)}
$$

and this is right! One can indeed find three different linear relations among the syzygies involving 4 column indices (the interested reader will find this easy), and with the right tools the proof that these generate all the relations is not too difficult. In fact the rest of the resolution is given by repeating this pattern until the binomial coefficient becomes 0 , giving

$$
\begin{aligned}
0 \rightarrow T(-q)^{(q-1)\left(\begin{array}{l}
q \\
q
\end{array}\right)} \rightarrow \cdots & \rightarrow \\
T(-3)^{2\left(\begin{array}{c}
q \\
3
\end{array}\right)} \rightarrow T(-2)^{\left(\begin{array}{c}
q \\
2
\end{array}\right)} & \rightarrow T \rightarrow T_{X} \rightarrow 0 .
\end{aligned}
$$

This complex is called the Eagon-Northcott complex.

A $2 \times q$ matrix of rank one can be specified by giving the kernel, the image, and a nonsingular transformation between 1-dimensional spaces (multiplication by some nonzero scalar), so the space of all such matrices has (affine) dimension $q-1+1+1=$ $q+1$ and codimension $2 q-(q+1)=q-1$. The codimension comes out the same if we think of $X$ as a projective variety in projective space.

On the other hand, a scroll $\Sigma\left(d_{1}, \ldots, d_{e}\right)$ is given by the minors of a $2 \times q$ matrix with $q=\sum d_{i}$; it has (projective) dimension $e$ and is naturally embedded in a projective space of dimension $\sum d_{i}+e-1$, so it too has codimension $q-1$. We may say that it is obtained by specializing the generic matrix $M$ to the special matrix $M\left(d_{1}, \ldots, d_{e}\right)$. It follows from work of Macaulay 24] that the whole resolution 
specializes under these circumstances; it is equivalent to saying that the $\operatorname{ring} S_{X}$ is a Cohen-Macaulay ring, though we will not need this point of view.

The main point that the reader should notice here is this: not only are the relations on the generators of the ideal defining a scroll linear (as Joe Harris had occasion to use) but the relations on these, the relations on those relations, and so on-all the "higher syzygies" - are also linear. This is what I noticed in Joe's lecture and what I hoped might be of use for the problem Joe was interested in. But... it was not. Mark Green actually solved the problem (the generation of the ideals of canonical curves by quadrics of rank four) a few years later in a different way (see Green [19]). This was disappointing but didn't diminish my interest in the subject, and Joe and I were luckier in other projects.

\section{What ABOUt REDUCIBLE SETS?}

Somehow this didn't decrease my enthusiasm for scrolls either, and I was happy to find lots of problems "left for me". One that caught my attention was, "What condition on more general algebraic sets (or even schemes) would be 'like' minimal degree?"

It will be important for what is coming to know that the condition "The ideal is generated by quadrics and the higher syzygies are all linear" holds not just for the scrolls, treated above, but for all varieties of minimal degree. We can check this using the classification. It is trivial for quadric hypersurfaces: the resolutions have the form

$$
0 \rightarrow S(-2) \rightarrow S \rightarrow S_{X} \rightarrow 0
$$

and there are no higher syzygies. Besides the scrolls and the quadric hypersurfaces, we need to check only the Veronese surface and the cones over it. These all correspond to the ideal generated by the $2 \times 2$ minors of a generic symmetric matrix. Since this is just one example, one can compute the resolution on a computer program such as Macaulay2 25, and the result has the form

$$
0 \rightarrow S(-4)^{3} \rightarrow S(-3)^{8} \rightarrow S(-2)^{6} \rightarrow S \rightarrow S_{X} \rightarrow 0 .
$$

Of course one can also give a conceptual proof.

Failure of the Basic Inequality. It's not obvious that it even makes sense to ask about reducible algebraic sets of minimal degree. After all, we know that Theorem 1 simply becomes false if we replace "variety" by "algebraic set".

The same failure carries over to the basic inequality of Corollary 2, which defines the notion of "minimal degree". First, of course, we must decide how to define the "degree" of a general algebraic set. The "right" choice for many contexts is that the degree of $X$ is the sum of the degrees of the components of $X$ having maximal dimension (the dimension of $X$ ), so let's adopt that for the moment. Unfortunately this has the consequence that the union of a line and 1000 points in $\mathbf{P}^{r}$ has degree 1. This set may easily span $\mathbf{P}^{r}$ and fail Corollary 2 .

We could hope that this failure comes just from the fact that our definition of degree ignores the lower-dimensional components, and try to build them in. If that were the problem, then things should be better when we look at equidimensional sets, that is, algebraic sets all of whose components have the same degree. But the union of two skew lines in $\mathbf{P}^{3}$ does not satisfy the basic inequality either, since $2 \nsupseteq 1+[3-1]$. Similarly, the union of two disjoint planes of dimension $n$ in $\mathbf{P}^{2 n+1}$ spans $\mathbf{P}^{2 n+1}$ but has degree $2 \nsupseteq 1+[(2 n+1)-n]$. 
On the other hand, reducible curves in the plane, such as the union of two lines, trivially do satisfy Corollary 2. The union of two lines in the plane is connected, while the union of two disjoint lines in space is not. Would connectedness help? Yes, as one easily checks, for curves. But no in general: the union of two two-planes in $\mathbf{P}^{4}$, meeting in a point, shows: $2 \nsupseteq 1+[4-2]$.

Another case where the basic inequality holds is the case where $X$ is a finite set of points. This is just linear algebra: a set of $d$ points cannot span a plane of dimension larger than $d-1$. Note that in this case the algebraic set is disconnected, so one might think that the truth of the basic inequality here comes from some completely separate principle.

Though they may seem quite different, the cases of points and of connected curves are both covered by the following definition: we say that an algebraic set $X$ is connected in codimension 1 if the irreducible components of $X$ all have the same dimension and can be ordered $X_{1}, \ldots, X_{k}$ so that each $X_{i} \cap X_{i+1}$ is of codimension 1 in $X$ (we regard the empty set as having dimension -1 ).

The proof of Corollary 2 extends easily to this case:

Corollary 7. If $X \subset \mathbf{P}^{r}$ is an algebraic set that is connected in codimension 1 , then $\operatorname{deg} X \geq 1+\operatorname{dim} \operatorname{span} X-\operatorname{dim} X$. For example, if $X$ spans $\mathbf{P}^{r}$, then $\operatorname{deg} X \geq$ $1+\operatorname{codim} X$.

This suggests that one might try, as a first step, to extend Theorem 5 to the case of algebraic sets that are connected in codimension 1. I suggested this problem to a student, Sebastian Xambó, and he was able to carry it out [31. The answer is pleasingly simple, involving just unions of scrolls in addition to the varieties of minimal degree.

But there is something unsatisfying in this. To exaggerate a bit (and with no negative reflection on what Xambó did): this is the case where things should work out "the same as before". The setting smells too much of a technique for generating results that I have always called "proof analysis": see what the previous researcher really proved and relax the hypotheses of his theorem a little, but not so much that his proof, or a little variation of it, won't still prove the result. There is a sense in which the case of the two skew lines in $\mathbf{P}^{3}$ is quite different from the irreducible case. Wouldn't it be nice to have a version of Theorem 5 that would at least say something about skew lines?

\section{Introduction to Castelnuovo-Mumford Regularity}

A little after this I did some work with Shiro Goto [12] on resolutions that are "like" the Eagon-Northcott complex. Among other matters, we studied ideals that are generated by quadratic forms and have linear resolutions, in the sense above; we called such ideals "2-linear". In fact, the homogeneous ideal of two skew lines $X$ in $\mathbf{P}^{3}$, which is (in suitable coordinates)

$$
(x, y) \cap(u, v)=(x, y) \cdot(u, v)=(x u, x v, y u, y v),
$$

is 2-linear; its resolution may be written:

$$
0 \rightarrow S \stackrel{\left(\begin{array}{l}
x \\
y \\
u \\
v
\end{array}\right)}{\longrightarrow} S^{4}(-3) \stackrel{\left(\begin{array}{cccc}
0 & v & 0 & -y \\
0 & -u & y & 0 \\
-v & 0 & 0 & x \\
u & 0 & -x & 0
\end{array}\right)}{\longrightarrow} S(-2)^{4} \stackrel{\left(\begin{array}{llll}
x u & x v & y u & y v
\end{array}\right)}{\longrightarrow} S \rightarrow S_{X}
$$


If, as in this case, the first map has quadratic entries, we say the resolution is "2-linear". Goto and I showed that such notions fit well with a somewhat opaque cohomological measure of complexity of a variety (or scheme) $X$, the CastelnuovoMumford regularity, or simply regularity of $X 5$ A special case of what we proved could be taken as a definition:

The regularity of $X$ is 2 (or $X$ is "2-regular") if and only if, when we regard $X$ as a subvariety of its linear span, the ideal of $X$ is 2-linear.

Goto and I saw from this criterion that varieties of minimal degree are 2-regular. We managed to prove a converse:

Theorem 8. If $X \subset \mathbf{P}^{r}$ is a variety then $X$ is 2-regular if and only if $X$ has minimal degree.

This raises the question of whether "2-regularity" could be the right condition to replace "minimal degree" in the case of reducible varieties. But of course it's not clear that there's any meaningful geometry in this condition!

We observed that one case beyond varieties of minimal degree is easy to analyze: the case of an algebraic set consisting of a finite number of points.

Proposition 9. An algebraic set consisting of a finite number of points is 2-regular if and only if the points are linearly independent.

Thus 2-regularity is a direct generalization of linear independence. The fact that among varieties 2-regularity characterizes "minimal degree" suggests that it might be interesting in general. Further evidence came from work of Fröberg [17, who classified the square-free monomial ideals that have 2-linear resolutions. In geometric terms this is a classification of the unions of coordinate planes (of various dimensions) that are 2-regular. The general case, given below, is not really more complicated to describe, so we will not pursue Fröberg's result.

\section{Smallness AND REgularity}

Though the definition of 2-regularity for an algebraic set $X$ is so ungeometric, Proposition 9 shows that the notion has a simple geometric meaning in the very special case where $X$ is a finite set. It seems natural to try to explore 2-regular sets in general by some reduction to this finite case, perhaps by taking general plane sections.

It would be nice if taking hyperplane sections somehow preserved the form of a free resolution, but it does not - even the length of a minimal resolution can change. If there is a sequence of hyperplanes $H_{1}, \ldots, H_{\operatorname{dim} X}$ such that

$$
Y=H_{1} \cap \cdots \cap H_{\operatorname{dim} X} \cap X
$$

is a finite set of points and the resolution of the ideal of $Y$ is obtained from that of $X$ by factoring out the equations of the $H_{i}$, then the homogeneous coordinate ring of $X$ is said to be Cohen-Macaulay. A result of Hartshorne (see [11, Theorem 18.12]) implies that in this case $X$ is connected in codimension 1 . So this idea can't be used to study 2-regular algebraic sets beyond the case treated by Xambó.

There is a way around this problem that produces a geometric property of 2-regular algebraic sets (and even schemes). It was hidden in plain sight for years

\footnotetext{
5 "Regularity" may well be the most overloaded word in mathematics!
} 
until it was elucidated by Jessica Sidman, a student of Lazarsfeld, in 30] and independently discovered, in a somewhat different way, by Giulio Caviglia [3. I kicked myself when I finally saw it: it's an easy variant of an argument that I had studied and admired and had thought I understood from a beautiful paper of Gruson, Lazarsfeld and Peskine [20. In the form relevant to this story, it says that you can forget transversality in the argument above as long as you are content to work only with 0 -dimensional plane sections of the algebraic set $X$. You don't get as strong a result: there's no reason to think that the form of the free resolution of the plane section has much similarity to that of $X$ - even its length can be different. But 2-regularity is preserved! (See Eisenbud, Green, Hulek and Popescu 13. and Eisenbud, Huneke and Ulrich [16] for various refinements, proven with other methods.)

Thus Proposition 9 implies an important geometric property of all 2-regular algebraic sets (and, more generally, schemes):

Proposition 10 (Sidman-Caviglia). If $X \subset \mathbf{P}^{r}$ is a 2-regular algebraic set, then for any linear space $L \subset \mathbf{P}^{r}$ such that $L \cap X$ is a finite set, the number of points of $L \cap X$, counted with multiplicity, is at most $1+\operatorname{dim} L$.

For convenience, we make the conclusion of this proposition into a definition:

Definition 11. If $X \subset \mathbf{P}^{r}$ is an algebraic set (or scheme), then $X$ is called small if, for any linear space $L \subset \mathbf{P}^{r}$ such that $L \cap X$ is a finite set, the number of points of $L \cap X$, counted with multiplicity, is at most $1+\operatorname{dim} L$.

With this language, Proposition 10 says simply that 2-regularity implies smallness.

This statement implies a more structural version: when $L \cap X$ is finite, then its points are linearly independent. The reason we have not stated Proposition 10 in terms of linear independence is that, to get the maximum value from it, we would have to interpret the linear independence scheme-theoretically, and the counting above does this for us without having to refer to the idea of a scheme.

For example, if $L$ is a line tangent to $X$ at a point $p$ and meeting $X$ additionally in a point $q$, then the number of points of intersection, counted with multiplicity, is at least $3>1+\operatorname{dim} L=2$, so $X$ could not be 2 -linear. To interpret this as a linear dependence statement, we have to recognize that the union of a double point and a separate point on a line is not linearly independent (the double point includes the whole line in its linear span).

Part of the power of Proposition 9 comes from the fact that we can choose linear spaces $L$ satisfying the hypothesis that are nevertheless very far from being transverse. For example, if $X$ is a 2-regular irreducible curve, not a line, in any projective space, the result shows that no line can meet $X$ more than 2 times.

For this reason smallness for varieties is, a priori, a stronger condition than minimal degree (which refers only to intersections with planes of complementary dimension). But by Theorem 8 varieties of minimal degree are 2-regular, so for varieties, smallness is actually equivalent to 2-regularity.

To sum up what we have done: we now have two conditions, smallness and 2regularity, that make sense for algebraic sets (and even schemes) and extend the notion of minimal degree for varieties. Smallness is a nice geometric condition. The condition of 2-regularity is a priori much stronger but inscrutably algebraic.

Small Algebraic Sets. Let's pause to describe two concrete examples. 
Example: Let $X=L_{1} \cup L_{2} \subset \mathbf{P}^{3}$ be the union of two disjoint lines in $\mathbf{P}^{3}$ (Figure 9). We exhibited the free resolution associated to $X$ at the beginning of Section 6, so we know that $X$ is 2-regular and thus, by Proposition 10, small. We can see this smallness directly: Let $L$ be a linear space. Clearly $L \cap X$ is finite unless $L$ contains one of the lines $L_{i}$, so we suppose this is not the case. If $L$ has dimension 0 , so $L$ is a point, then it is clear that $L \cap X$ is at most 1 point. If $L$ is a line or a plane, then $L$ can meet each $L_{i}$ in at most 1 point (with multiplicity), so the number of intersections with $X$ is at most $2 \leq 1+\operatorname{dim} L$.

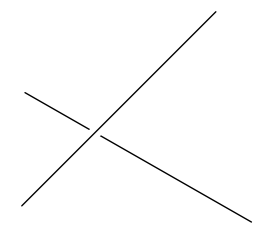

Figure 9. Skew lines in 3-space.

Example: More interesting is the case $X=L_{1} \cup L_{2} \cup L_{3} \subset \mathbf{P}^{4}$, where the $L_{i}$ are general lines (Figure 10). A linear space $L \subset \mathbf{P}^{4}$ not containing any of the lines can meet each one in at most a point, as before, so the total number of intersections with $X$ is at most 3. Thus a 2-plane or 3-plane must meet $X$ in a way that is consistent with smallness. What about lines meeting $X$ ? Could there be one meeting $X$ three times?

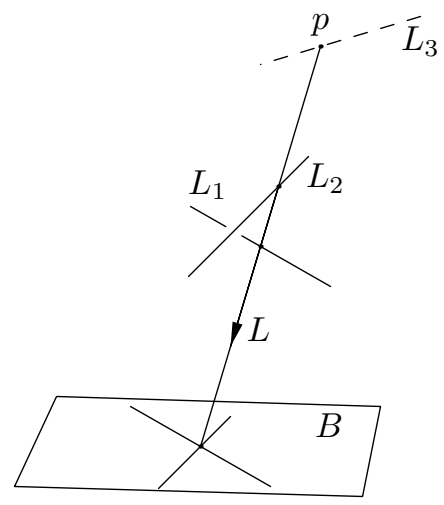

FiguRE 10. Three lines in 4-space always have a trisecant line.

In fact there is such a trisecant line, so $X$ is not small! Let $A$ be the linear space spanned by the skew lines $L_{1}, L_{2}$, so that $A$ is a 3 -plane, a hyperplane in $\mathbf{P}^{4}$. The line $L_{3}$ meets $A$ in a point $p$. If we project $A$ from $p$ onto a 2-plane $B$, the two lines $L_{1}$ and $L_{2}$ project to lines in $B$, and thus their projections meet (in just one point). Back in $A$ this means that there is a (unique) line $L$ through $p$ that meets both $L_{1}$ and $L_{2}$. In fact this line $L$ is the unique linear space in $\mathbf{P}^{4}$ that violates the condition of smallness for $X$ !

Of course this implies that $X$ is not 2-regular. On the other hand it turns out that $Y=X \cup L$ is 2-regular. This can easily be checked on a computer, but it will follow immediately from the construction principle to which we now turn. 


\section{LiNEARLY JOINED SEQUENCES OF VARIETIES}

How can one produce 2-regular algebraic sets to play with? By Theorem 5] and Theorem 8 we know all the 2-regular irreducible varieties, so it is natural to ask whether we can stick these together in some simple way to form more complicated examples. A little experimentation with exact sequences shows that if $X$ and $Y$ are 2-regular algebraic sets (or even schemes) such that the intersection of $X$ and $Y$ is the same as the intersection of their linear spans,

$$
X \cap Y=\operatorname{span}(X) \cap \operatorname{span}(Y),
$$

then $X \cup Y$ is again 2-regular.

Note that the condition $(* *)$ on $X \cap Y$ implies in particular that $X \cap Y$ is a linear space. Of course $(* *)$ is much stronger: for example, two conics meeting in one point in $\mathbf{P}^{3}$ do not satisfy $(* *)$, because the span of each is a plane, and these planes meet in a line.

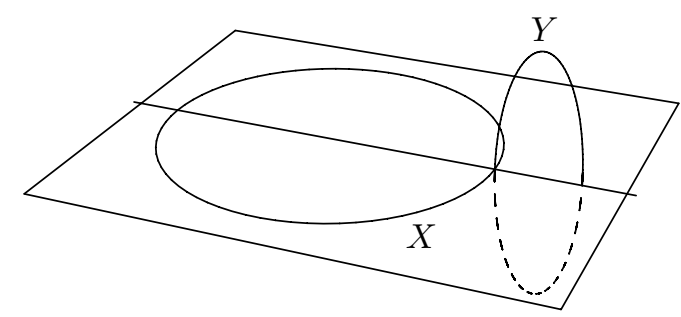

Figure 11. Two conics in 3-space meeting in a point are not linearly joined.

Proceeding inductively, we are led to make the following definition:

Definition 12. A sequence $\left(X_{1}, \ldots, X_{s}\right)$ of varieties (or algebraic sets or schemes) in a projective space is linearly joined if for each $i$,

$$
\left(X_{1} \cup \cdots \cup X_{i}\right) \cap X_{i+1}=\operatorname{span}\left(X_{1} \cup \cdots \cup X_{i}\right) \cap \operatorname{span}\left(X_{i+1}\right) .
$$

Using the idea above, we get an inductive method for constructing 2-regular algebraic sets (or schemes):

Corollary 13. If $\left(X_{1}, \ldots, X_{s}\right)$ is a linearly joined sequence and each $X_{i}$ is 2regular, then the union $X_{1} \cup \cdots \cup X_{s}$ is 2-regular.

Although algebraic sets that are constructed as the union of linearly joined sequences of varieties of minimal degree come with a sort of ready-made structure theorem, the notion is not so straightforward as it may appear. For example, the order of the $X_{i}$ in the definition above is important, as the example in Figure 12 shows.

In any event, we get a third notion extending the definition of minimal degree from varieties to algebraic sets: a union of a linearly joined sequence of varieties of minimal degree. This notion

- is a priori even stronger than 2-regularity,

- is quite geometric,

- has a structure theorem

but is not very intrinsic and somehow (to my taste) not very elegant. 


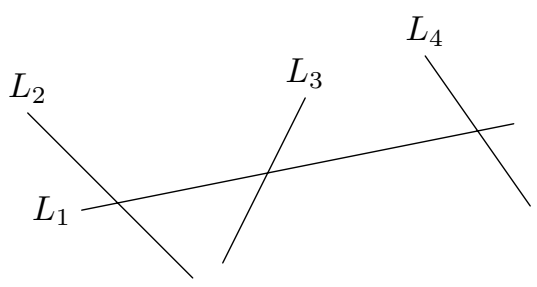

Figure 12. $\left\{L_{1}, L_{2}, L_{3}, L_{4}\right\}$ is a linearly joined sequence, but $\left\{L_{2}, L_{3}, L_{4}, L_{1}\right\}$ is not.

\section{Classification of small algebraic sets}

When we had reached this point, Sorin Popescu and I each spent a lot of time looking at examples on the computer. It's hard to see how to check smallness directly, but 2-regularity is perfectly suited for computation (we used the program Macaulay 2 [25]), and linearly joined sequences of minimal degree varieties are relatively easy to spot. So we looked for 2-regular examples that were not linearly joined sequences of this kind. No luck.

Could this mean that the two notions were equivalent? Von Neumann says somewhere that the real use of computers in mathematics will be to improve the intuition of mathematicians. I would add that the data produced by computation frequently lead to conjectures and (often) to strong convictions about them 6

Despite the need for caution, many good ideas have come from such experiments; I myself owe some of my best theorems to data collected by "playing" with Macaulay, and then Macaulay2, specialized programs for symbolic computation in projective geometry and commutative algebra. This inductive method of discovering mathematical theorems provides a nice complement to the deductive method, which might (somewhat uncharitably) be described as piling theorems one already knows on top of each other to guess and make a new theorem. The inductive method often leads to conjectures that are trivial, or merely uninteresting, and sometimes to conjectures that are very hard because they come without a pedigree of the reasons for believing in them. But sometimes it leads to conjectures having a kind of freshness and originality that may be harder to achieve with the deductive method.

Perhaps the ease and tradition of inductive work is the reason number theory has so many more wonderful - and difficult — conjectures than other fields of mathematics. I think many of these other fields are gaining, as computer experiments become easier and more popular.

\footnotetext{
${ }^{6}$ One must be a little suspicious. Probably the most famous example concerns the prime number theorem $\pi(x) \sim \operatorname{Li}(x)$, where $\pi(x)$ is the number of primes less than $x$ and

$$
\operatorname{Li}(x)=\int_{2}^{x} \frac{d x}{\ln (x)}
$$

If you compute both sides for lots of values of $x$, first by hand and then on a machine, you will notice that in every example there is an inequality $\pi(x)<L i(x)$. Both Gauss and Riemann were led by such experiments to conjecture that this inequality holds for every $x$. Nevertheless, G. H. Hardy showed that the difference $\pi(x)-L i(x)$ changes sign infinitely often. It turns out that the first $x$ for which this sign reversal occurs is well beyond what currently imaginable computation could find!
} 
In any case, the computations that Sorin and I made gave us a conviction that for algebraic sets, the three potential extensions of the notion of minimal degree were in fact equivalent. Our faith was a little weakened, though, by the fact that, unlike nearly everything else I've described so far, the equivalence definitely does not hold for schemes; the computer provided us with many such examples, of which more below.

Despite bits and pieces of progress toward the conjecture made in joint work with Klaus Hulek, we were, for several years, unable to decide the question. Was the conjecture just a result of ignorance coming from our failure to point the computers in the right direction?

In the early spring of 2003, Green, Hulek, Popescu and I were all able to get together in Banff in the program supporting "Research in Teams". There are such programs at Oberwolfach and Luminy too, and they are often undersubscribed. People find it hard to get away, and certainly it wasn't easy for the four of us. But the time was idyllic: we worked most of the day and took a long chilly walk together through that inspiring landscape in the afternoon. The reward of being able to focus all our attention on our problem was crucial progress. Although the "proof" we produced had to be repaired later, the basic ideas held. I highly recommend this kind of experience!

Here's the result:

Theorem 14 (Eisenbud, Green, Hulek and Popescu [13]). If $X \subset \mathbf{P}^{r}$ is an algebraic set, the following are equivalent:

1) $X$ is small.

2) $X$ is 2-regular.

3) $X$ is the union of a linearly joined sequence of varieties of minimal degree.

Since, as we have indicated, implications 3$) \Rightarrow 2$ ) $\Rightarrow 1$ ) hold for all schemes, this yields:

Corollary 15. If an ideal is 2-regular, then its radical is 2-regular.

The corollary surprised me partly because a natural generalization, the statement that the radical of an ideal has no larger regularity than the ideal itself, is false (see (4).

\section{ABIDing Mysteries}

When you arrive, out of breath, at the mountaintop, you get a new view of the world. Deeply satisfying - at least temporarily. The new view often includes new tops. Perhaps they seem even higher than the one on which you stand, or perhaps they look like they have interesting rocks or another desirable view, or perhaps you just want the pleasure of another struggle and completion. A new project is often born in the death of an old one. Here's a sample of one question coming from the work above, which Sorin Popescu and I have been discussing.

Schemes in General. What do 2-regular schemes look like? I'll assume in this last section that the reader is familiar with the theory of schemes in projective space (it's just the theory of saturated homogeneous ideals in a polynomial ring).

I hope for a description, not a classification; indeed, I think no one will ever classify 2-regular schemes. This is because every finite scheme can be embedded in projective space as a 2-regular scheme (this is like the statement that any abstract 
set can be embedded as a linearly independent set of vectors in some vector space). Now a finite subscheme of a projective space is the same thing as a finite-dimensional commutative algebra $A$ plus a subspace of $A$ containing the identity element, such that the subspace generates $A$ as an algebra. The finite subscheme is 2-regular if and only if the subspace is all of $A$. But already the finite-dimensional commutative algebras are in an important sense unclassifiable.

If a description is what we want, we must ask what interesting properties 2regular schemes could share. We've already seen that if $X$ is 2-regular, then its reduced part $X_{\text {red }}$ is a 2-regular algebraic set. Here's my favorite conjecture in this matter as of January 2007; I've been able to verify it only in some rather special cases.

Conjecture 16. If $X$ is a 2-regular scheme, then the inclusion $X_{\text {red }} \rightarrow X$ splits, and the ideal of $X_{\text {red }}$ in $X$ is 1-regular.

\section{IS THERE A MORAL?}

So what about the question of how problems are chosen. One of my favorite stories is by Feynman, who tells of his sense of gratitude when he first learned that there were some problems that were not yet solved: "Something left for me." Once, as president of the AMS, I got to ask all the members of the Council to tell of something that contributed to their becoming mathematicians. There were stories of mentors and of inspiring, or aggravating, books. There were surprisingly many stories of some obstacle overcome, something that looked scary and hard but was accomplished.

Perhaps, gentle reader, like Feynman, you'll be pleased to know there's plenty left for you!

\section{AbOut THE AUTHOR}

David Eisenbud is the director of the Mathematical Sciences Research Institute and a professor at the University of California, Berkeley. He is past president of the AMS. This article is based on his Retiring Presidential Address given in San Antonio in 2006.

\section{REFERENCES}

1. Bertini, E., Introduzione alla Geometria Proiettiva degli Iperspazi. Enrico Spoerri, Pisa (1907). Freely available from Cornell University Library, http://historical.library.cornell.edu/.

2. Buchsbaum, D. A., Lectures on regular local rings. 1969 Category Theory, Homology Theory and Their Applications, I (Battelle Institute Conference, Seattle, Wash., 1968, Vol. One), pp. 13-32, Springer, Berlin. MR0244243 (39:5560)

3. Caviglia, G., Koszul Algebras, Castelnuovo-Mumford Regularity, and Generic Initial Ideals. PhD Thesis, University of Kansas, 2004.

4. Chardin, M., and D'Cruz, C., Castelnuovo-Mumford regularity: examples of curves and surfaces. J. Algebra 270 (2003), no. 1, 347-360. MR2016666 (2004m:13036)

5. Conca, Aldo, Straightening law and powers of determinantal ideals of Hankel matrices. Adv. Math. 138 (1998), no. 2, 263-292. MR.1645574 (99i:13020)

6. Demazure, M., and Gabriel, P., Groupes algébriques. Tome I: Géométrie algébrique, généralités, groupes commutatifs. Avec un appendice Corps de classes local par Michiel Hazewinkel. Masson \& Cie, Éditeur, Paris; North-Holland Publishing Co., Amsterdam, 1970. MR0302656 (46:1800) 
7. Eagon, J. A., and Northcott, D. G., Ideals defined by matrices and a certain complex associated with them. Proc. Roy. Soc. Ser. A 269 (1962), 188-204. MR0142592 (26:161)

8. Eisenbud, D., Subrings of Artinian and Noetherian rings. Math. Ann. 185 (1970), 247-249. MR 0262275 (41:6885)

9. Eisenbud, D., Transcanonical embeddings of hyperelliptic curves. J. Pure Appl. Algebra 19 (1980), 77-83. MR593247 (82f:14027)

10. Eisenbud, D., Linear sections of determinantal varieties. Amer. J. Math. 110 (1988), no. 3, 541-575. MR944327 (89h:14041)

11. Eisenbud, D., Commutative Algebra with a View toward Algebraic Geometry. Springer-Verlag, New York (1995). MR1322960 (97a:13001)

12. Eisenbud, D., and Goto, S., Linear free resolutions and minimal multiplicity. J. Algebra 88 (1984), no. 1, 89-133. MR741934 (85f:13023)

13. Eisenbud, D., Green, M., Hulek, K., and Popescu, S., Small Schemes and Varieties of Minimal Degree. http://front.math.ucdavis.edu/math.AG/0404517 (to appear in the American Journal of Mathematics).

14. Eisenbud, D., Green, M., Hulek, K., and Popescu, S., Restricting linear syzygies: algebra and geometry. Compos. Math. 141 (2005), no. 6, 1460-1478. MR2188445 (2006m:14072)

15. Eisenbud, D., and Harris, J., On varieties of minimal degree (a centennial account). Algebraic geometry, Bowdoin, 1985 (Brunswick, Maine, 1985), 3-13, Proc. Sympos. Pure Math., 46, Part 1, Amer. Math. Soc., Providence, RI, 1987. MR.927946 (89f:14042)

16. Eisenbud, D., Huneke, C., and Ulrich, B., The regularity of Tor and graded Betti numbers. Amer. J. Math. 128 (2006), no. 3, 573-605. MR2230917 (2007b:13027)

17. Fröberg, R., Rings with monomial relations having linear resolutions. J. Pure Appl. Algebra 38 (1985), no. 2-3, 235-241. MR814179 (87b:13020)

18. Gallego, F. J., and Purnaprajna, B., Triple canonical covers of varieties of minimal degree. A tribute to C. S. Seshadri (Chennai, 2002), 241-270, Trends Math., Birkhäuser, Basel, 2003. MR2017587 (2005f:14033)

19. Green, M. L., Quadrics of rank four in the ideal of a canonical curve. Invent. Math. 75 (1984), no. 1, 85-104. MR728141 (85f:14028)

20. Gruson, L., Lazarsfeld, R., and Peskine, C., On a theorem of Castelnuovo, and the equations defining space curves. Invent. Math. 72 (1983), no. 3, 491-506. MR704401 (85g:14033)

21. Hartshorne, R., Algebraic geometry. Graduate Texts in Mathematics, No. 52, SpringerVerlag, New York-Heidelberg, 1977. MR0463157 (57:3116)

22. Hauser, H., http://www1-c703.uibk.ac.at/mathematik/project/bildergalerie/gallery.html

23. Mac Lane, S., Saunders Mac Lane-A Mathematical Autobiography. With a preface by David Eisenbud. A K Peters, Ltd., Wellesley, MA, 2005. MR2141000 (2006b:01010)

24. Macaulay, F. S., The Algebraic Theory of Modular Systems. Revised reprint of the 1916 original. With an introduction by Paul Roberts. Cambridge Mathematical Library, Cambridge University Press, Cambridge, 1994. MR 1281612 (95i:13001)

25. Grayson, D., and Stillman, M., Macaulay 2, a software system for research in algebraic geometry, available at http://www.math.uiuc.edu/Macaulay2/.

26. Mumford, D., Algebraic Geometry. I. Complex Projective Varieties. Reprint of the 1976 edition. Classics in Mathematics, Springer-Verlag, Berlin, 1995. MR1344216 (96d:14001)

27. del Pezzo, P., Sulle superficie di ordine $\mathrm{n}$ immerse nello spazio di $\mathrm{n}+1$ dimensioni, Rend. Circ. Mat. Palermo 1 (1886).

28. Rathmann, J., The uniform position principle for curves in characteristic $p$. Math. Ann. 276 (1987), no. 4, 565-579. MR879536 (89g:14026)

29. Room, T. G., The Geometry of Determinantal Loci. Cambridge Univ. Press, 1938.

30. Sidman, J., On the Castelnuovo-Mumford regularity of products of ideal sheaves. Adv. Geom. 2 (2002), no. 3, 219-229. MR.1924756 (2003f:13021)

31. Xambó, S., On projective varieties of minimal degree. Collect. Math. 32 (1981), no. 2, 149-163. MR 653892(84e:14039)

Mathematical Sciences Research Institute, 17 Gauss Way, Berkeley, California 94720-5070; Department of Mathematics, University of California, Berkeley, CaliforNIA 94720 